Rapid Reviews COVID-19

\title{
Review 1: "Ultrasensitive and Selective Detection of SARS-CoV-2 Using Thermotropic Liquid Crystals and Image-Based Machine Learning"
}

\section{Samuel Lalmuanawma ${ }^{1}$}

${ }^{1}$ Mizoram University, Mathematics \& Computer Science, India

Published on: Nov 22, 2020

DOI: 10.1162/2e3983f5.cabe00b8

License: Creative Commons Attribution 4.0 International License (CC-BY 4.0). 


\section{$\underline{\text { RR:C19 Evidence Scale rating by reviewer: }}$}

- Strong. The main study claims are very well-justified by the data and analytic methods used. There is little room for doubt that the study produced has very similar results and conclusions as compared with the hypothetical ideal study. The study's main claims should be considered conclusive and actionable without reservation.

$* * * * * * * * * * * * * * * * * * * * * * * * * * * * * * * * * * * * * * * *$

\section{Review:}

When it comes to clinical diagnosing, significant attention has been given to the application of the LC biosensor due to its unique property of physicochemical and microstructures. The preprint applied the same process for an ultrasensitive and selective detection of the SARS-CoV-2 virus for a point-of-care diagnostic device on an Android mobile phone base.

The overall method and approach are valid with a high-quality standard, the research was timely and highly significant, and the research interest extremely high during the current pandemic situation. The authors concentrated on lengthy and quality results with discussion ranging from the preparation of the film till the final test results read out by the smart phone application.

In the preprint, they mention that "a partially self-assembled monolayer of cationic surfactants was formed at an aqueous-LC interface, followed by the adsorption of a 15mer ssDNA probe with LC interface". It tried to demonstrate that the ordering transition in the formed LC surface strongly depends on the targeted nucleotide sequence. Furthermore, the authors designed an LC-based SARS-CoV-2 RNA point-ofcare detection kit, with an obtained response that is visible to the naked eye without any additional equipment, and a smart phone-based application.

However, the current preprint version does not contain certain brief and clear pieces of information that are required to be included in the publication. Readers may benefit from a discussion of the following points:

1. Accuracy of the proposed model was not compared (specificity and sensitivity) with the RT-PCR, which is still now a gold standard for clinical diagnosis and the authors need to state what the main advantages will be compared to the existing model/system. 
2. Validation of the proposed LC model for SARS-CoV-2 detection must also be based on standard specificity at $100 \%$.

3. Definition or assay of system specification for smart phone (Camera specs. like sensor, pixels, processor speed, etc. recommended by the proposed model) will be highly significant for the readers.

4. The molecules of LC were extremely subjective to the arrangement of its surface alignment, a tiny adjustment on its surface leads the molecules of LC to be oriented in a diverse course and results in some transmitted light intensity through its cell when observed in a polarizing optical microscope. This means the factor-like intensity of the light etc. may affect the results and, consequently, the assumptions of error probability, cost, and management respectively. 